\title{
Protocolo de seguimiento para proyectos de restauración forestal
}

\author{
Monitoring protocol for forest restoration projects \\ Cuevas Wizner, R. '; Oliet Palá, J.A.'; Melero de Blas, M.* \\ ${ }^{1}$ Departamento de Sistemas y Recursos Forestales. E.T.S. de Ingeniería de montes, forestal y del medio natural. \\ Universidad Politécnica de Madrid. Paseo de las Moreras s/n, 28040, Madrid \\ ${ }^{2}$ WWF España. Gran Via de San Francisco, 8D, 28005, Madrid
}

Autor para correspondencia: mmelero@wwf.es 


\title{
Resumen
}

WWF España, en colaboración con la Universidad Politécnica de Madrid, ha desarrollado un protocolo de seguimiento para proyectos de restauración forestal. El objetivo ha sido estandarizar las técnicas para garantizar un seguimiento a corto y medio plazo de la vegetación, optimizando la eficiencia del esfuerzo de muestreo para mantener una precisión determinada. Las variables principales que se proponen para la vegetación introducida son: estado vegetativo y supervivencia, y variables morfológicas (altura, diámetro de la base del tronco o diámetro de copa del matorral). De forma complementaria, se recomienda contabilizar otras variables cuantitativas de la vegetación espontánea (número de especies, cobertura y talla). Basándose en los trabajos de seguimiento previos realizados por WWF, se han fijado tamaños de muestra tanto para las variables morfológicas, como para las variables cualitativas. Asimismo, se introduce el concepto de "Especie Principal Menos Representada" (EPMR) que será aquella a la que se dirigirá el error de muestreo admisible. En el protocolo propuesto se recomienda agrupar los individuos muestreados en un diseño a base de parcelas circulares. La diferencia en la intensidad de muestreo entre variables morfológicas y la supervivencia se resuelve utilizando dos tamaños de parcelas concéntricas. Además, para el muestreo de la vegetación espontánea se propone un diseño superpuesto al anterior, con parcelas cuadradas. Finalmente, se recomienda realizar un muestreo inicial, tras la ejecución de la repoblación, y un muestreo post-establecimiento, en el primer otoño, sugiriéndose un tercero al año y, con una frecuencia en descenso, a partir de los años siguientes.

Palabras clave: evaluación, muestreo, parcelas, repoblación, variables.

\begin{abstract}
WWF Spain, in collaboration with 'Universidad Politécnica de Madrid', has developed a monitoring protocol for forest restoration projects. The aim has been to standardize techniques that ensure short- and medium-term monitoring of vegetation, optimizing efficiency of the sampling effort to maintain an adequate precision. Main variables proposed for introduced vegetation are: vegetative state and survival, and morphological variables (height, diameter of the base of the trunk or bush diameter for scrubs). It is also recommended to take into account other quantitative variables of native vegetation (number of species, coverage and height). Based on previous monitoring works carried out by WWF, sample sizes have been set for both morphological variables and qualitative variables. The concept of "Least Represented Main Species" is introduced as well, that be the one to which the admissible sampling error will be directed. In the proposed protocol, it is recommended to group sampled individuals in a design with circular plots. The difference in sampling intensity between morphological variables and survival is solved using two sizes of concentric plots. In addition, for the sampling of native vegetation, a design superimposed on the previous one is proposed, with square plots. Finally, it is recommended to carry out an initial sampling, after the implementation of the reforestation, and a post-establishment sampling, in the first autumn, suggesting a third after a year and, with a decreasing frequency, from the following years.
\end{abstract}

Keywords: assessment, sampling, plots, reforestation, variables. 


\section{Introducción}

El seguimiento es una labor de interés creciente en el ámbito de la restauración forestal: por una parte, permite aprender de los errores y aciertos de la ejecución de la técnica repobladora, al poderse asociar el fallo o el éxito de una actuación con la técnica de ejecución; y por otro, permite extraer información muy valiosa sobre los efectos a futuro que tiene la repoblación sobre los sistemas en restauración.

Sin embargo, el análisis de la situación de la restauración forestal en España durante los últimos años (Cuenca, 2014; Colomina y Melero, 2016; Cuenca et al., 2016) constató que una de las principales carencias corresponde precisamente a la realización de labores de seguimiento y evaluación post proyecto. Aunque estas labores generalmente se contemplan, su consideración está escasamente planificada y frecuentemente depende de cada proyecto concreto. El único criterio utilizado de forma generalizada en el seguimiento de la plantación es la supervivencia de la planta, olvidando otros aspectos clave como el cumplimiento de los objetivos previstos y los efectos sociales, económicos y ambientales de las actividades ejecutadas. Una de las causas de esta carencia puede estar relacionada con la falta de herramientas técnicas de evaluación adaptadas específicamente a este propósito.

WWF España tiene entre sus prioridades la restauración de espacios forestales degradados, que entiende esencial para luchar contra la desertificación, la fragmentación de los hábitats y el cambio climático. En este sentido, la organización ha desarrollado desde 1989, más de 30 proyectos en ecosistemas degradados necesitados de una restauración prioritaria, tales como zonas incendiadas de alto valor de conservación, hábitats de alto interés ecológico como ríos o riberas, o enclaves relevantes para la recuperación de especies relictas. WWF lleva realizando el seguimiento de sus proyectos de restauración forestal desde el año 2009. La experiencia adquirida en este campo ha puesto de manifiesto la importancia de realizar un seguimiento adaptado a este tipo de proyectos.

A partir de las premisas anteriores, se ha visto necesario elaborar una metodología de inventario y de evaluación de plantaciones adaptada a las características de los proyectos y basada en la optimización de la eficiencia del esfuerzo de muestreo. Así este protocolo pretende establecer una estandarización en las técnicas y criterios de evaluación para garantizar un seguimiento de calidad de los proyectos de restauración de WWF a lo largo del tiempo.

Los resultados que se vayan obteniendo con la metodología propuesta aportarán nueva información y experiencia para el seguimiento, lo cual retroalimentará el protocolo propuesto. De igual manera, se espera que éste pueda ser utilizado por otras entidades privadas o públicas para valorar el éxito de sus restauraciones dada la actual carencia detectada. 


\section{Material y métodos}

La presente publicación está basada en Cuevas (2018) y recoge un resumen de la propuesta que, de forma más pormenorizada, se discute en dicho trabajo.

El protocolo que se presenta está adaptado a las características de las repoblaciones ejecutadas por WWF, caracterizadas en su mayoría por una gran diversidad de especies (más de 5 especies), la baja densidad de cada una en plantación $(<200$ pies $\mathrm{ha}^{-1}$ ) y la abundancia de actuaciones en pequeñas superficies (menos de $10 \mathrm{ha}$ ). Asimismo, se dispone de bastante información inicial de cada proyecto, tanto del diseño como de la ejecución, lo cual ofrece oportunidades para ajustar el seguimiento a las particularidades de la zona a inventariar.

Este protocolo se ha orientado a realizar el seguimiento a corto plazo en relación con la vida de una restauración. No se han incluido por tanto aspectos que se deberían evaluar a medio y a largo plazo, como los cambios en las propiedades del suelo o en la complejidad y funcionalidad del ecosistema.

Los trabajos de seguimiento previos realizados por WWF, con ciertas variaciones entre proyectos, han supuesto un banco de información crucial de cara al diseño del muestreo. Por ello, este protocolo ha utilizado esta información en la determinación del número de plantas a medir (intensidad de muestreo) para conseguir una estimación fiable de la población, aspecto fundamental en el diseño de un muestreo.

Dado que la realización de muestreos piloto es una tarea costosa, en este protocolo se han empleado los datos de los muestreos realizados por WWF en proyectos de restauración previos. Con esta información se ha ajustado un modelo de regresión para variables cuantitativas que ha permitido establecer el número de individuos que es necesario muestrear de manera que se asegure un error máximo admisible. Así se han tomado 90 casos de muestreo y se han ajustado las curvas potenciales para el diámetro en la base del tronco y para la altura, relacionando tamaños de muestra con el error relativo que se obtenía. De igual forma, en el caso de variables cualitativas, se ha realizado un ejercicio teórico, construyendo una gráfica con diferentes tamaños de población, relacionando el tamaño de muestra necesario para obtener diferentes errores absolutos. En todos los casos se ha establecido un 30\% de error relativo máximo admisible con una probabilidad fiducial del $95 \%$, valor utilizado habitualmente en inventarios de tipo forestal no productivo (Oliet et al., 2005).

Otros aspectos del diseño del muestreo se han basado en las técnicas tradicionales de inventario, aunque particularizando para los aspectos específicos del recurso a inventariar.

\section{Resultados y discusión}

Se presenta a continuación la descripción de la metodología de muestreo que se propone en el protocolo: 


\subsection{Variables inventariadas}

El protocolo centra el diseño del seguimiento en variables asociadas a la vegetación introducida, de mayor interés, ya que son la principal herramienta con la que se realiza la restauración. No obstante, interesa saber asimismo cómo reacciona la vegetación espontánea para poder valorar a largo plazo la recuperación del sistema y la evolución de la biodiversidad. Por ello, el protocolo establece una metodología para el inventario y seguimiento de la vegetación espontánea, con unas exigencias de muestreo menores y un procedimiento diferente que para la implantada (Fig. 1).

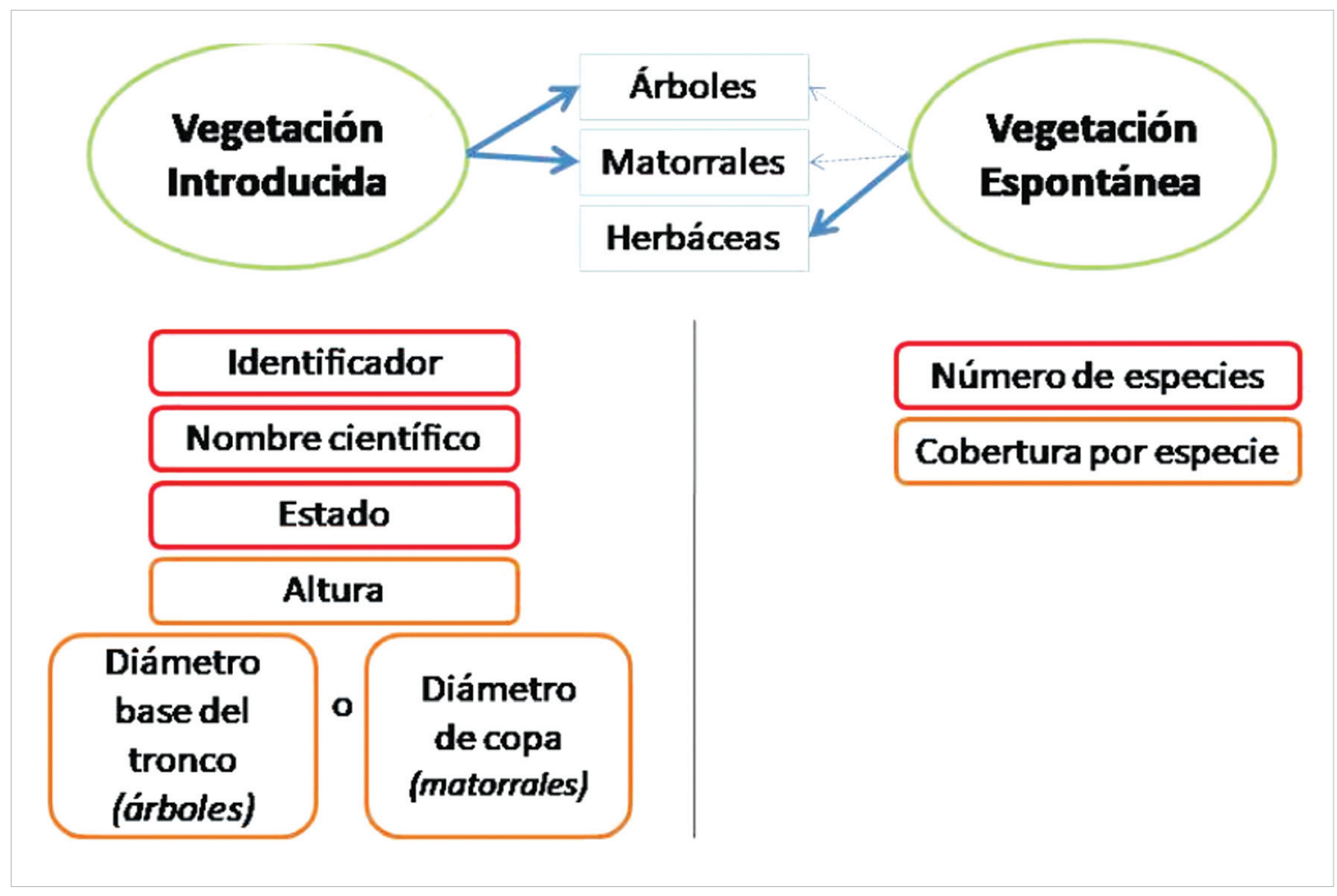

Figura 1. Esquema de las variables de la vegetación según el tipo (Cuevas, 2018)

\subsubsection{Vegetación introducida}

Las variables que se deben tener en cuenta para el inventario de la vegetación introducida serán de dos tipos: cualitativas (aquellas características que describen un atributo y son clasificadas en tipos) y cuantitativas (cuando pueden ser medidas y cuantificadas).

\section{Cualitativas.}

Estado vegetativo. Se debe llevar un control sobre las plantas que están vivas y las que han muerto. 


\section{Cuantitativas.}

Diámetro a la base del tallo. Es una variable muy importante, dado que está relacionada simultáneamente con el desarrollo de la parte aérea y radical del árbol. En arbolitos jóvenes como los que son objeto de seguimiento, el diámetro debe medirse en la base del tallo principal, aquel de mayor altura. La medición debe hacerse a ras de suelo, aunque evitando engrosamientos anómalos. Conviene marcar el lugar de medición para poder repetir la medida en el mismo lugar en futuros muestreos. Es conveniente el uso de un calibre con precisión de $0,1 \mathrm{~mm}$ dado el orden de magnitud de los tamaños en estas etapas. Conviene realizar dos medidas perpendiculares y considerar la media de los resultados, para evitar medidas sesgadas por errores de medición o asimetrías del tallo.

Altura. Es otra variable importante para valorar el grado de desarrollo de las plantas. Se debe medir la altura verde, es decir, la distancia proyectada sobre un eje vertical desde la base del tallo, donde está la marca anteriormente mencionada para el diámetro, hasta el punto donde se encuentre la yema u hoja viva más elevada de la planta. Para los órdenes de magnitud de esta variable se necesita una escala de precisión de $1 \mathrm{~cm}$.

Diámetro de la copa de matorral. En el caso del matorral no es posible diferenciar un tallo principal debido a las múltiples ramificaciones que aparecen en la base. Como alternativa para obtener una variable que esté relacionada con el desarrollo de la parte aérea, se propone la medición del diámetro de copa como sustituto de la medida del diámetro de la base del tronco. Se debe medir la proyección ortogonal sobre el suelo de la copa en dos diámetros perpendiculares entre los que debe estar la dimensión máxima y considerar la media de los resultados, para evitar medidas sesgadas por errores de medición o asimetrías del tallo. La precisión a utilizar en este caso será de $1 \mathrm{~cm}$.

\subsubsection{Vegetación espontánea}

La vegetación espontánea se muestreará registrando las siguientes variables:

Número de especies. Establecer el número de especies presentes en una parcela puede ser un objetivo asequible y permite obtener un indicador de diversidad vegetal específico sencillo (riqueza florística). Se debe tratar de identificar o diferenciar las especies concretas. Cuando esto no sea posible, se debe aproximar taxonómicamente al máximo (género, familia), y si esto no es posible, se recomienda al menos diferenciar entre herbáceas y leñosas.

Cobertura. Se debe hacer una estimación conjunta del porcentaje cubierto del suelo por el estrato herbáceo y de matorral. Esta variable da una indicación 
del grado de protección del suelo y de los procesos hidrológicos como la infiltración o la escorrentía. Además, para cada una de las especies, se debe asignar una cobertura, lo cual permitirá obtener indicadores ecológicos más precisos y completos. Este protocolo propone el empleo de una escala simplificada de Braun-Blanquet.

Talla. Para conocer el grado de competencia y el desarrollo de la vegetación espontánea herbácea y leñosa, se estimará para cada especie la talla o altura media del conjunto de individuos de cada parcela, realizando una medición vertical con cinta métrica y utilizando una precisión en intervalos de 10 $\mathrm{cm}$. Esta medición será complementaria a la de cobertura y nos dará una idea de la ocupación espacial de la vegetación competidora y sus posibles efectos sobre las plantas introducidas.

\subsection{Tamaños muestrales}

En el caso de variables cualitativas como la supervivencia, se ha optado por una aproximación teórica para fijar el tamaño de muestra inicial. Para ello se ha construido una gráfica con diferentes tamaños de población, relacionando el tamaño de muestra necesario para obtener diferentes errores absolutos (Fig. 2). Así, el esfuerzo necesario para disminuir el error absoluto aumenta drásticamente en torno a un error del 0,25 . Tomando los valores para diferentes tamaños de población, se observa que utilizar aproximadamente 30 individuos garantiza un error absoluto inferior a $15 \%$ en poblaciones pequeñas, que equivale al error relativo máximo admisible fijado del $30 \%$.

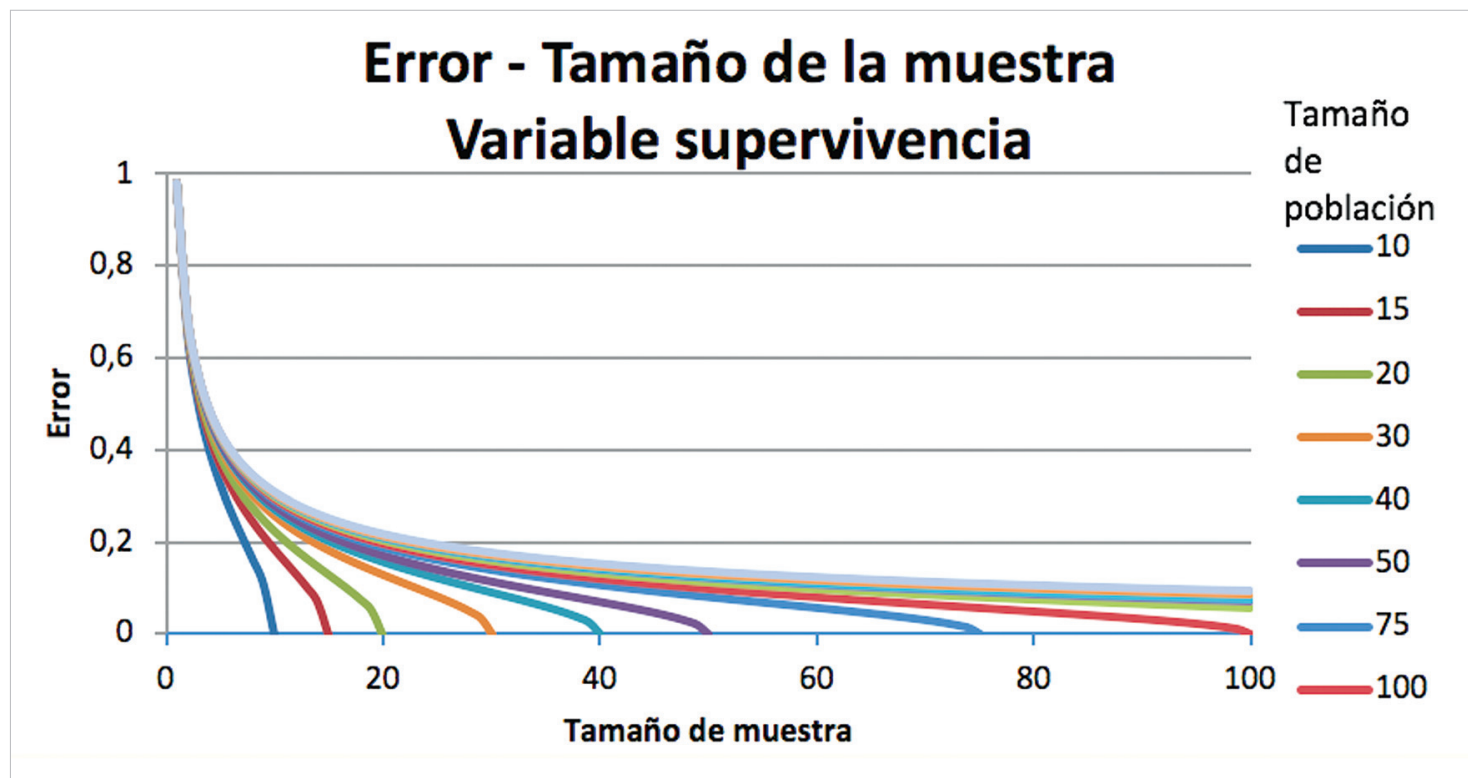

Figura 2. Relación del error absoluto y el tamaño de muestra para diferentes tamaños de población, con una varianza del 0,25 y nivel de confianza del 95\% (Cuevas, 2018) 
Para las variables cuantitativas y fruto de los análisis de regresión realizados con los seguimientos que WWF ha llevado a cabo en sus proyectos pasados, se ha observado que se requieren aproximadamente 8 individuos para el diámetro en base del tronco (Fig. 3) y 7 individuos para la altura (Fig. 4), ya que por encima de estas intensidades de muestreo no se mejora significativamente la precisión con respecto al umbral prefijado del $30 \%$ de error relativo. Basándose en estas estimaciones, este protocolo fija, para un primer muestreo en el que la variabilidad es desconocida, un tamaño de muestra de 10 individuos para una especie como número práctico para asegurar ese error relativo del $30 \%$ en diámetro y altura.

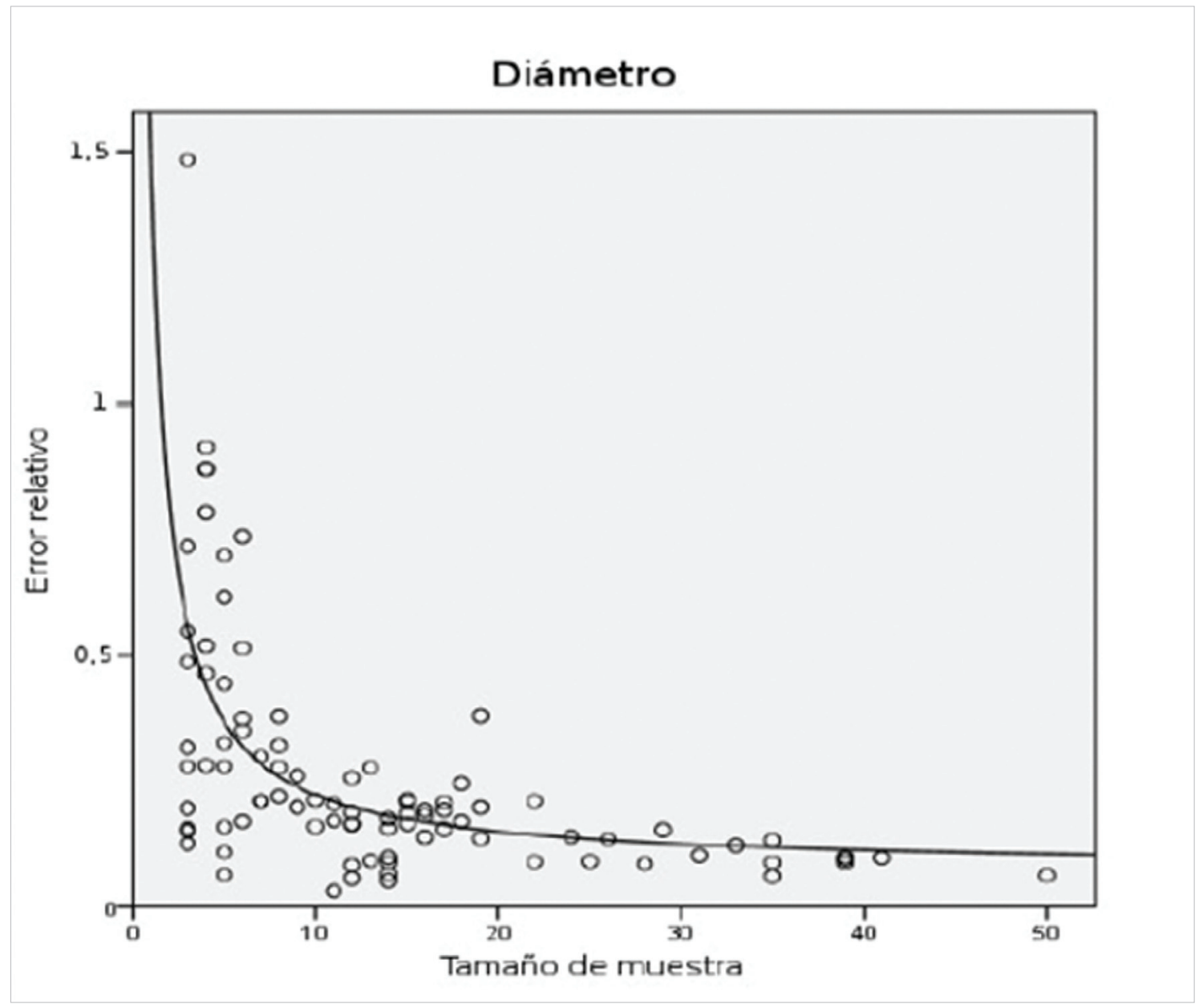

Figura 3. Relación del tamaño muestra y error relativo para el diámetro de tronco (Hermosilla, 2016).

Cada especie introducida puede tener una densidad de plantación diferente, siendo necesario muestrear una superficie diferente para obtener el mismo número de individuos, lo cual no sería práctico. Para salvar esta circunstancia se propone fijar una especie objetivo para la que se dimensiona el tamaño del muestreo. Así se introduce el concepto de especie principal menos representada (EPMR), como 


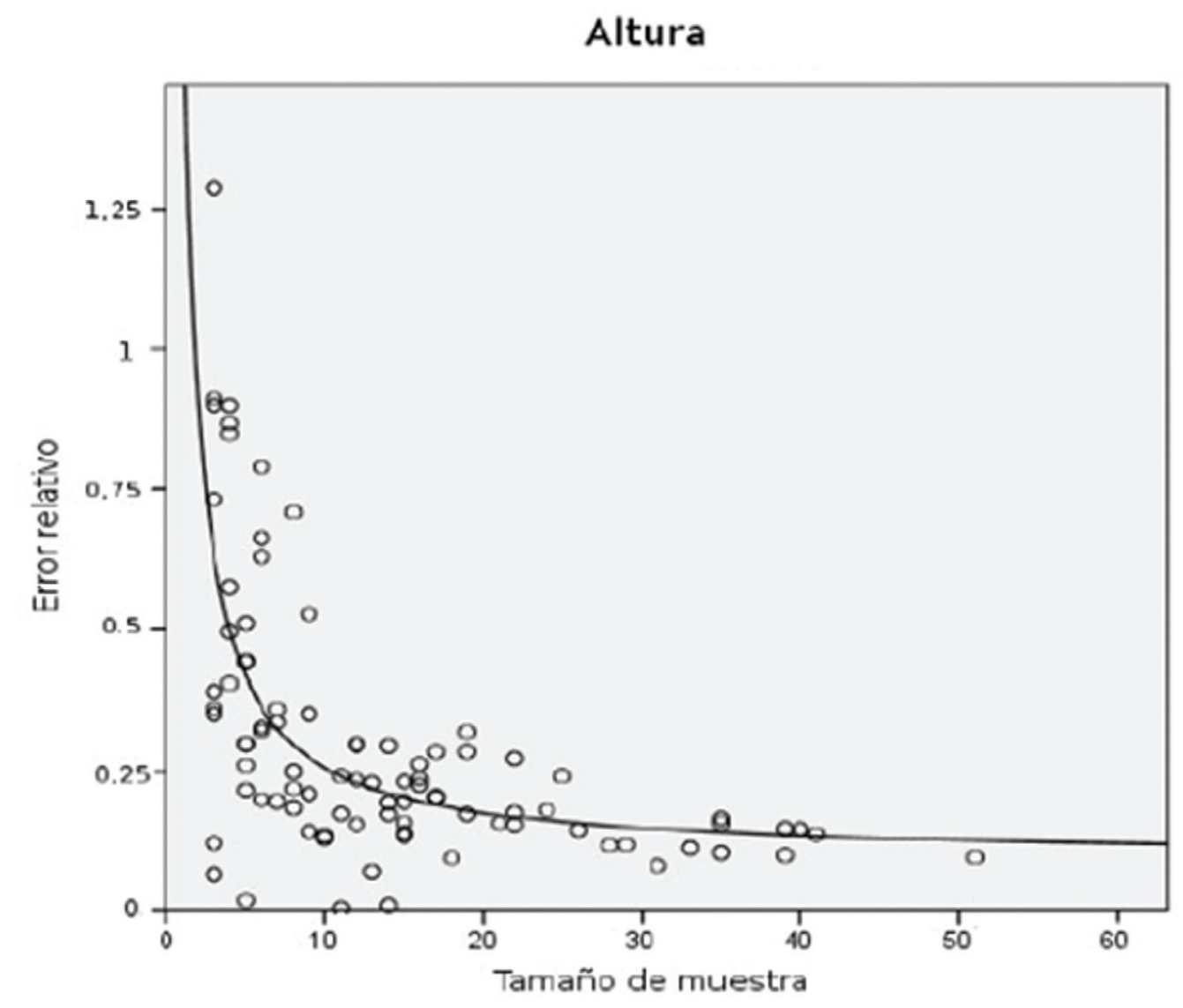

Figura 4. Relación del tamaño muestra y error relativo para la altura (Hermosilla, 2016).

la especie que, dentro de las introducidas en la repoblación, tiene una abundancia mínima a la que dirigir el error de muestreo admisible. Para las especies con mayor densidad de plantación que la EPMR se obtendrán más individuos de los necesarios, disminuyendo el error y, para las especies con menor densidad de plantación (y por tanto de menor importancia relativa) que la EPMR, se obtendrán menos individuos de los necesarios, superando el error admisible.

La EPMR deberá cumplir los siguientes requisitos:

- El número de individuos deberá representar al menos la proporción correspondiente al inverso del número de especies, para que tenga una importancia suficiente en el ámbito de la actuación.

- El número de plantas no deberá ser inferior al 50\% del número de plantas de la especie con mayor representación, garantizando que la especie más representada no se muestree en exceso, siendo posible que la EPMR coincida con la especie más representada cuando sea la única que cumpla el criterio. 
- Una densidad mínima entre 50 y 100 pies/ha en función de la superficie de la actuación, que garantice que el porcentaje de superficie de muestreo no sea excesiva.

\subsection{Diseño del muestreo}

El rodal de repoblación es la unidad que se utiliza en el diseño de repoblaciones para identificar zonas donde se aplicará el mismo sistema de repoblación, entendiendo por tal la coincidencia del mismo método de preparación del terreno (que incluye tanto el procedimiento de eliminación de la vegetación competidora como la preparación del suelo), las mismas especies elegidas y su método de repoblación (siembra o plantación) y un idéntico diseño de plantación. Por lo general, el rodal de repoblación habrá sido ejecutado simultáneamente en una misma campaña de plantación.

Es la unidad más apropiada para adoptar como sujeto del seguimiento, dado que tiene características homogéneas internamente y por lo tanto los datos que obtenemos de la muestra podremos extrapolarlos al resto del rodal. El rodal de repoblación será por tanto la unidad de referencia para fijar las solicitudes del muestreo (error admisible para un nivel de confianza), constituyendo la unidad inventarial.

La estimación de las características del rodal se realizará a partir de una muestra de éste. La unidad muestral será la parcela. Las parcelas de muestreo son áreas delimitadas que incluyen las plantas que se medirán. Este sistema de agrupación en parcelas es el que se empleará siempre, tanto para la vegetación introducida como para la espontánea, dado que ofrece mayores ventajas prácticas.

Hay tres aspectos a tener en cuenta para definir la parcela: forma, superficie y distribución.

\subsubsection{Forma y tamaño de las parcelas}

Las formas más habituales de las parcelas de muestreo son circulares, cuadradas o rectangulares.

Las parcelas circulares tienen menor coste de replanteo ya que sólo necesitan las coordenadas del centro y el radio. El borde, al ser equidistante del centro, no incurre en sesgo por la orientación y además se minimiza el "efecto borde". El mayor inconveniente de este tipo de parcelas es la dificultad para la delimitación exacta de sus límites.

Las parcelas cuadradas minimizan su perímetro frente a las parcelas rectangulares, disminuyen el efecto borde y por tanto son más adecuadas para vegetación herbácea. Para este tipo de vegetación un límite recto ayuda a separar con mayor claridad las plantas que pertenecen a la parcela.

Por las razones expuestas, se utilizarán las parcelas circulares para la vegetación introducida, y parcelas cuadradas para la vegetación espontánea.

En el caso de la vegetación introducida, se considerará que un plantón está incluido en la parcela cuando la planta esté efectivamente dentro del perímetro, quedando excluida si solo queda incluido el alcorque. 
Por su parte, para la vegetación espontánea, se utilizarán parcelas cuadradas, recomendándose un tamaño de $25 \mathrm{~m}^{2}$ (inferior a lo que recomienda la literatura; Mue1ler-Dombois and Ellemberg, 1974).

El diseño de muestreo quedará entonces de la siguiente forma (Fig. 5):

- Una primera parcela circular (limitada en azul en la figura), donde se medirán las variables diámetro y altura de las plantas introducidas (pequeños círculos marrones en la figura) y cuyo radio (R1) dependerá de la densidad de la EPMR y el número de parcelas.

- Una segunda parcela circular (limitada en rojo en la figura) con una superficie tres veces superior a la anterior $(R 2=R 1 \sqrt{ } 3)$ donde se evaluará la supervivencia de las plantas introducidas (pequeños círculos marrones en la figura).

- Y, por último, una parcela cuadrada (limitada en verde en la figura) de $5 \mathrm{~m}$ de lado (L), donde se contarán el número de especies de la vegetación espontánea, su cobertura y talla.

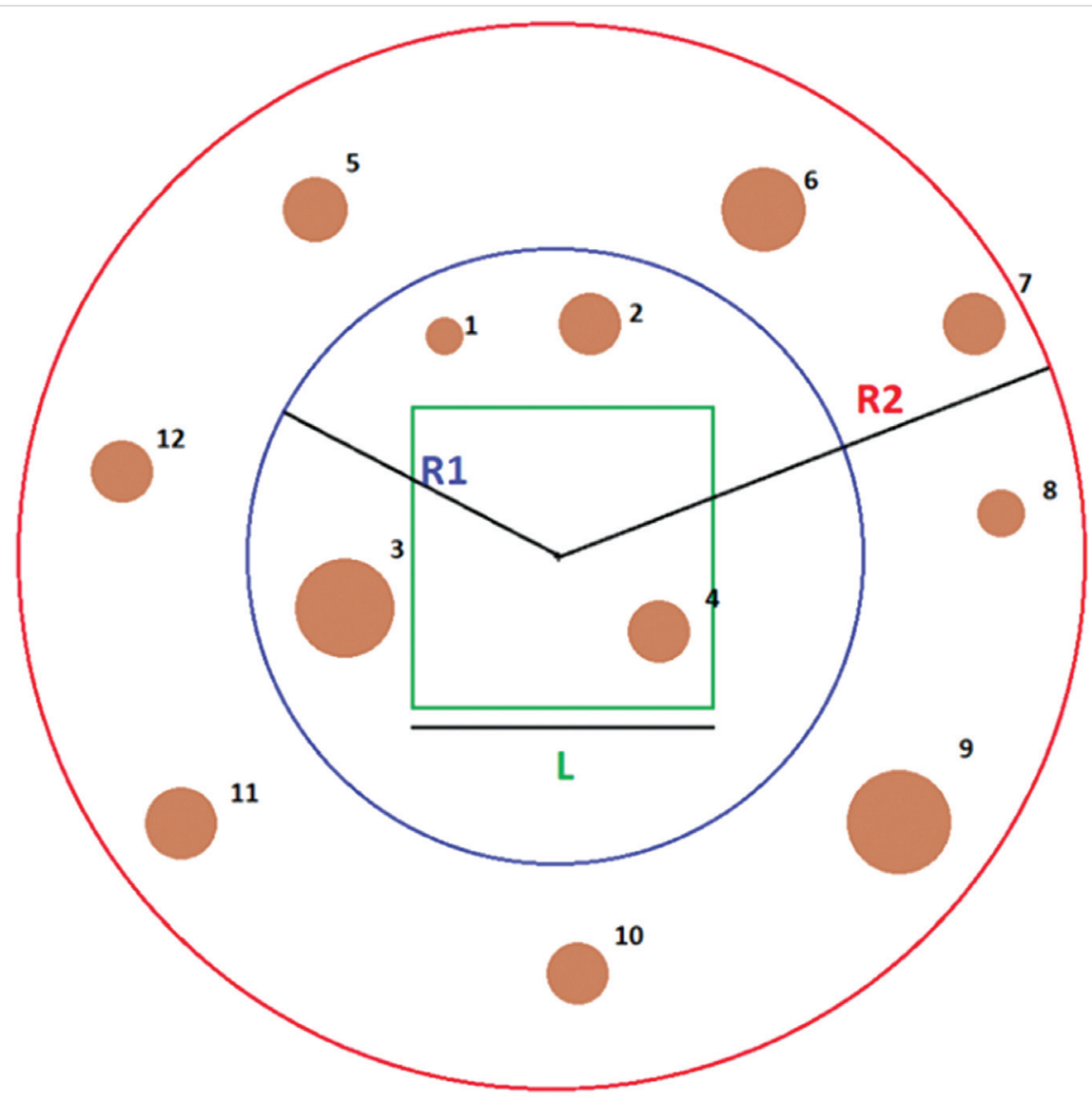

Figura 5. Parcelas concéntricas utilizadas en el muestreo (Cuevas, 2018). 


\begin{tabular}{|c|c|c|c|c|c|c|c|c|c|c|c|}
\hline 고 & & 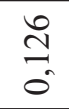 & $\begin{array}{l}\vec{n} \\
\\
0\end{array}$ & $\hat{\hat{n}}$ & $\begin{array}{l}\text { ñ } \\
\tilde{n} \\
\tilde{0}\end{array}$ & 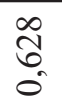 & 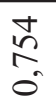 & $\begin{array}{l}0 \\
\infty \\
\infty \\
0\end{array}$ & $\stackrel{8}{0}$ & $\vec{\approx}$ & $\stackrel{n}{n}$ \\
\hline 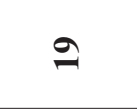 & & $\stackrel{n}{\tilde{0}}$ & 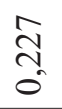 & 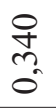 & 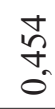 & 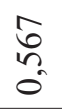 & $\begin{array}{l}8 \\
\infty \\
0 \\
0 \\
0\end{array}$ & $\frac{\pi}{\pi}$ & $\begin{array}{l}\hat{\delta} \\
\hat{\sigma}\end{array}$ & $\overrightarrow{\widetilde{O}}$ & $\stackrel{\Xi}{=}$ \\
\hline$\stackrel{\infty}{\sim}$ & & $\frac{N}{0}$ & $\begin{array}{l}\text { I্ } \\
\text { N. }\end{array}$ & $\begin{array}{l}n \\
\tilde{n} \\
0 \\
0\end{array}$ & \begin{tabular}{l}
$\hat{o}$ \\
\multirow{0}{*}{}
\end{tabular} & $\begin{array}{l}\stackrel{8}{0} \\
\stackrel{n}{0} \\
0\end{array}$ & $\begin{array}{l}7 \\
\overline{0} \\
0\end{array}$ & $\frac{n}{\tilde{\sigma}}$ & $\begin{array}{l}\underset{\infty}{+} \\
0 \\
0\end{array}$ & $\begin{array}{l}0 \\
\sigma \\
\sigma\end{array}$ & $\stackrel{\infty}{0}$ \\
\hline$\beth$ & & $\vec{a}$ & $\frac{\infty}{\infty}$ & $\stackrel{N}{\hat{N}}$ & $\begin{array}{l}\text { } \\
\tilde{n} \\
\tilde{0}\end{array}$ & 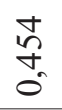 & $\begin{array}{l}n \\
\tilde{n} \\
\tilde{n}\end{array}$ & $\begin{array}{l}0 \\
\text { రై } \\
0 \\
0\end{array}$ & 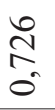 & $\begin{array}{l}\hat{D} \\
\infty \\
0\end{array}$ & $\begin{array}{l}\infty \\
\stackrel{\infty}{\circ} \\
\tilde{\sigma}\end{array}$ \\
\hline $\mathscr{0}$ & & $\stackrel{\infty}{8}$ & $\begin{array}{l}0 \\
0 \\
0\end{array}$ & 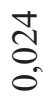 & हூ & సે & Oे & $\begin{array}{l}n \\
2 \\
0 \\
0\end{array}$ & $\begin{array}{l}\hat{\delta} \\
0 \\
0\end{array}$ & $\overrightarrow{\hat{\sigma}}$ & $\begin{array}{l}\stackrel{2}{\hat{0}} \\
0 \\
0\end{array}$ \\
\hline 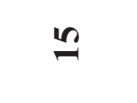 & & $\begin{array}{l}\infty \\
8 \\
0\end{array}$ & $\begin{array}{l}0 \\
0 \\
0\end{array}$ & $\begin{array}{l}\stackrel{J}{\Delta} \\
\underset{0}{0}\end{array}$ & $\begin{array}{l}\bar{n} \\
0 \\
0\end{array}$ & ڤે & $\begin{array}{l}\text { Oे } \\
0 \\
0\end{array}$ & $\begin{array}{l}n \\
2 \\
0 \\
0\end{array}$ & $\begin{array}{l}\text { ڤి } \\
0 \\
0\end{array}$ & $\stackrel{\overrightarrow{0}}{0}$ & $\begin{array}{l}\hat{\sigma} \\
0 \\
0\end{array}$ \\
\hline \pm & & $\begin{array}{l}\infty \\
8 \\
0 \\
0\end{array}$ & $\begin{array}{l}0 \\
0 \\
0\end{array}$ & 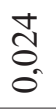 & $\begin{array}{l}\overline{0} \\
0\end{array}$ & ભે & $\underset{0}{\tilde{O}}$ & $\begin{array}{l}n \\
\tilde{o} \\
0\end{array}$ & $\begin{array}{l}\text { ֶे } \\
0 \\
0\end{array}$ & $\vec{\sigma}$ & 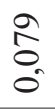 \\
\hline 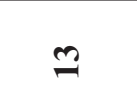 & & $\begin{array}{l}\infty \\
8 \\
0 \\
0\end{array}$ & $\begin{array}{l}0 \\
0 \\
0\end{array}$ & $\begin{array}{l}\stackrel{J}{\delta} \\
0 \\
0\end{array}$ & $\begin{array}{l}\overline{0} \\
0 \\
0\end{array}$ & के & Oे & $\begin{array}{l}n \\
2 \\
0 \\
0\end{array}$ & $\begin{array}{l}3 \\
0 \\
0 \\
0\end{array}$ & $\overrightarrow{\widehat{O}}$ & $\begin{array}{l}0 \\
\hat{0} \\
0\end{array}$ \\
\hline$\simeq$ & 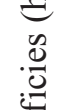 & $\begin{array}{l}\infty \\
8 \\
0\end{array}$ & $\begin{array}{l}0 \\
0 \\
0 \\
0\end{array}$ & $\begin{array}{l}\stackrel{J}{\Delta} \\
\text { O. }\end{array}$ & $\begin{array}{l}\bar{\sigma} \\
0 \\
0\end{array}$ & ڤે & $\begin{array}{l}\text { Oे } \\
0 \\
0\end{array}$ & $\begin{array}{l}n \\
\tilde{2} \\
0 \\
0\end{array}$ & $\begin{array}{l}\text { ชి } \\
0 \\
0\end{array}$ & $\vec{\sigma}$ & $\begin{array}{l}\hat{\sigma} \\
0 \\
0\end{array}$ \\
\hline$=$ & $\begin{array}{l}\overline{\bar{D}} \\
\stackrel{\Xi}{n}\end{array}$ & $\stackrel{\infty}{8}$ & $\begin{array}{l}0 \\
0 \\
0\end{array}$ & $\underset{0}{\stackrel{\Xi}{\sigma}}$ & है & ભે & Oे & $\begin{array}{l}n \\
\tilde{o} \\
0\end{array}$ & $\begin{array}{l}\Re \\
0 \\
0\end{array}$ & $\vec{\sigma}$ & $\begin{array}{l}2 \\
0 \\
0 \\
0\end{array}$ \\
\hline 으 & & $\begin{array}{l}\infty \\
8 \\
0 \\
0\end{array}$ & $\begin{array}{l}0 \\
0 \\
0\end{array}$ & 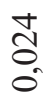 & $\begin{array}{l}\bar{\sigma} \\
0 \\
0\end{array}$ & ڤે & $\begin{array}{l}\text { Oे } \\
0\end{array}$ & $\begin{array}{l}n \\
2 \\
0 \\
0\end{array}$ & $\begin{array}{l}\text { ڤூo } \\
0 \\
0\end{array}$ & $\stackrel{\overrightarrow{0}}{0}$ & $\begin{array}{l}\hat{\sigma} \\
0 \\
0\end{array}$ \\
\hline$a$ & & $\begin{array}{l}\infty \\
8 \\
0\end{array}$ & $\begin{array}{l}0 \\
0 \\
0 \\
0\end{array}$ & $\begin{array}{l}\stackrel{J}{\Delta} \\
\text { Oे }\end{array}$ & 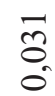 & $\begin{array}{c}\text { ले } \\
\text { Oे }\end{array}$ & $\begin{array}{l}\hat{J} \\
0 \\
0\end{array}$ & $\begin{array}{l}n \\
\tilde{o} \\
0 \\
0\end{array}$ & $\hat{\mathscr{b}}$ & $\overrightarrow{0}$ & $\begin{array}{l}\hat{\delta} \\
0 \\
0\end{array}$ \\
\hline$\infty$ & & $\begin{array}{l}\infty \\
8 \\
0 \\
0\end{array}$ & $\begin{array}{l}0 \\
0 \\
0 \\
0\end{array}$ & $\begin{array}{l}\stackrel{\Xi}{\Delta} \\
0 \\
0\end{array}$ & $\begin{array}{l}\bar{n} \\
0 \\
0\end{array}$ & ڤે & $\underset{0}{\tilde{O}}$ & $\begin{array}{l}n \\
2 \\
0 \\
0\end{array}$ & $\begin{array}{l}\hat{b} \\
0 \\
0\end{array}$ & $\stackrel{5}{0}$ & $\begin{array}{l}0 \\
\hat{0} \\
0\end{array}$ \\
\hline$r$ & & $\stackrel{\infty}{8}$ & $\begin{array}{l}0 \\
0 \\
0\end{array}$ & $\begin{array}{l}\stackrel{J}{\Delta} \\
\text { O. }\end{array}$ & $\overline{0}$ & ભે & $\underset{0}{O}$ & $\begin{array}{l}n \\
\tilde{o} \\
0\end{array}$ & $\begin{array}{l}\Re \\
0 \\
0\end{array}$ & $\underset{0}{\mathbb{0}}$ & $\begin{array}{l}\stackrel{2}{\hat{0}} \\
0 \\
0\end{array}$ \\
\hline b & & $\begin{array}{l}\infty \\
8 \\
0 \\
0\end{array}$ & $\begin{array}{l}0 \\
0 \\
0\end{array}$ & 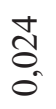 & $\begin{array}{l}\bar{\sigma} \\
0 \\
0\end{array}$ & ڤे & $\begin{array}{l}\hat{\sigma} \\
0\end{array}$ & $\begin{array}{l}n \\
2 \\
0 \\
0\end{array}$ & $\begin{array}{l}\text { ชి } \\
0 \\
0\end{array}$ & $\overrightarrow{0}$ & $\begin{array}{l}\stackrel{2}{\hat{0}} \\
0 \\
0\end{array}$ \\
\hline in & & $\begin{array}{l}\infty \\
\stackrel{8}{0} \\
0\end{array}$ & $\begin{array}{l}0 \\
0 \\
0 \\
0\end{array}$ & $\begin{array}{l}\stackrel{J}{\Delta} \\
\stackrel{0}{0}\end{array}$ & $\begin{array}{l}\bar{\sigma} \\
0 \\
0\end{array}$ & ڤે & $\underset{0}{\tilde{O}}$ & $\begin{array}{l}n \\
\tilde{o} \\
0 \\
0\end{array}$ & $\begin{array}{l}\hat{b} \\
0 \\
0\end{array}$ & $\vec{\delta}$ & $\begin{array}{l}\stackrel{2}{\hat{0}} \\
0 \\
0\end{array}$ \\
\hline 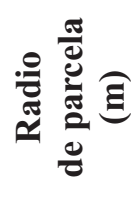 & & - & $N$ & $m$ & $\nabla$ & in & 0 & $r$ & $\infty$ & $a$ & 으 \\
\hline \multicolumn{12}{|c|}{ 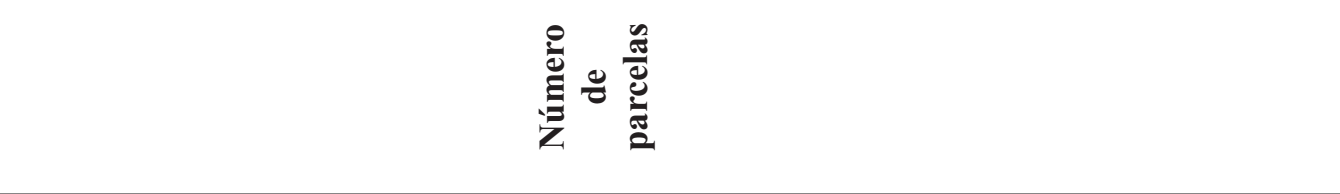 } \\
\hline
\end{tabular}


Para el caso de la vegetación introducida, se deberá elegir la combinación entre el tamaño de parcela con el número de ellas que cumpla con los requisitos del muestreo y maximice su eficiencia (Tab. 1). Se recomienda un tamaño de parcela que oscile entre 5 y $20 \mathrm{~m}$ de radio.

\subsubsection{Distribución de las parcelas}

Se empleará un muestreo sistemático (McRoberts et al., 2015), estableciéndose una malla de puntos de forma que se ubiquen todos los puntos de muestreo lo más separados posible de manera regular. Podremos utilizar diferentes patrones para establecer la malla, siendo los más comunes en malla cuadrada o rectangular, o a tresbolillo.

El número de parcelas para conseguir una representatividad adecuada dependerá de la homogeneidad del rodal de repoblación, aunque se puede considerar que con menos de cinco parcelas será muy difícil alcanzar una representatividad adecuada.

\subsubsection{Señalamiento del muestreo}

Las parcelas se localizarán a partir de las coordenadas de su centro, utilizando el sistema geodésico de referencia en España que según el RD 1071/2007 será ETRS89, coordenadas UTM. Posteriormente la localización de dichas parcelas se almacenará en un Sistema de Información Geográfica.

Se recomienda utilizar algún elemento físico sobre el terreno para que sea más fácil identificar donde se ha situado el centro de las parcelas. No debe ser muy llamativo para que no sea víctima de actos vandálicos. Dicho elemento también puede contar con algún tipo de identificador, para facilitar el muestreo y disuadir de su sustracción.

Puede ser recomendable el marcaje de las plantas muestreadas dentro de las parcelas lo que ayudará a determinar los límites de la parcela y la localización de los ejemplares. Se puede emplear un alambre rodeando el tallo, pero de manera holgada para que no anille la planta, o bien otras técnicas como el uso de etiquetas metálicas. Además, se puede marcar la sección basal del tallo en la cual se ha medido el diámetro, para que los seguimientos posteriores sean más exactos. El marcaje será importante en el caso de las sustituciones de marras. Para ello se deberá usar un identificador, como un alambre de otro color, siendo este color el mismo en todas las actuaciones.

\subsection{Frecuencia del seguimiento}

Según las etapas de un proyecto de repoblación, se han identificado y ubicado en el tiempo diversas actuaciones de seguimiento para cada una de las fases (Fig. 6).

\subsubsection{Caracterización de la repoblación}

Con esta actuación se pretende recoger los aspectos estáticos e invariables en el tiempo de la repoblación: localización, diseño, especies... Esta información se 


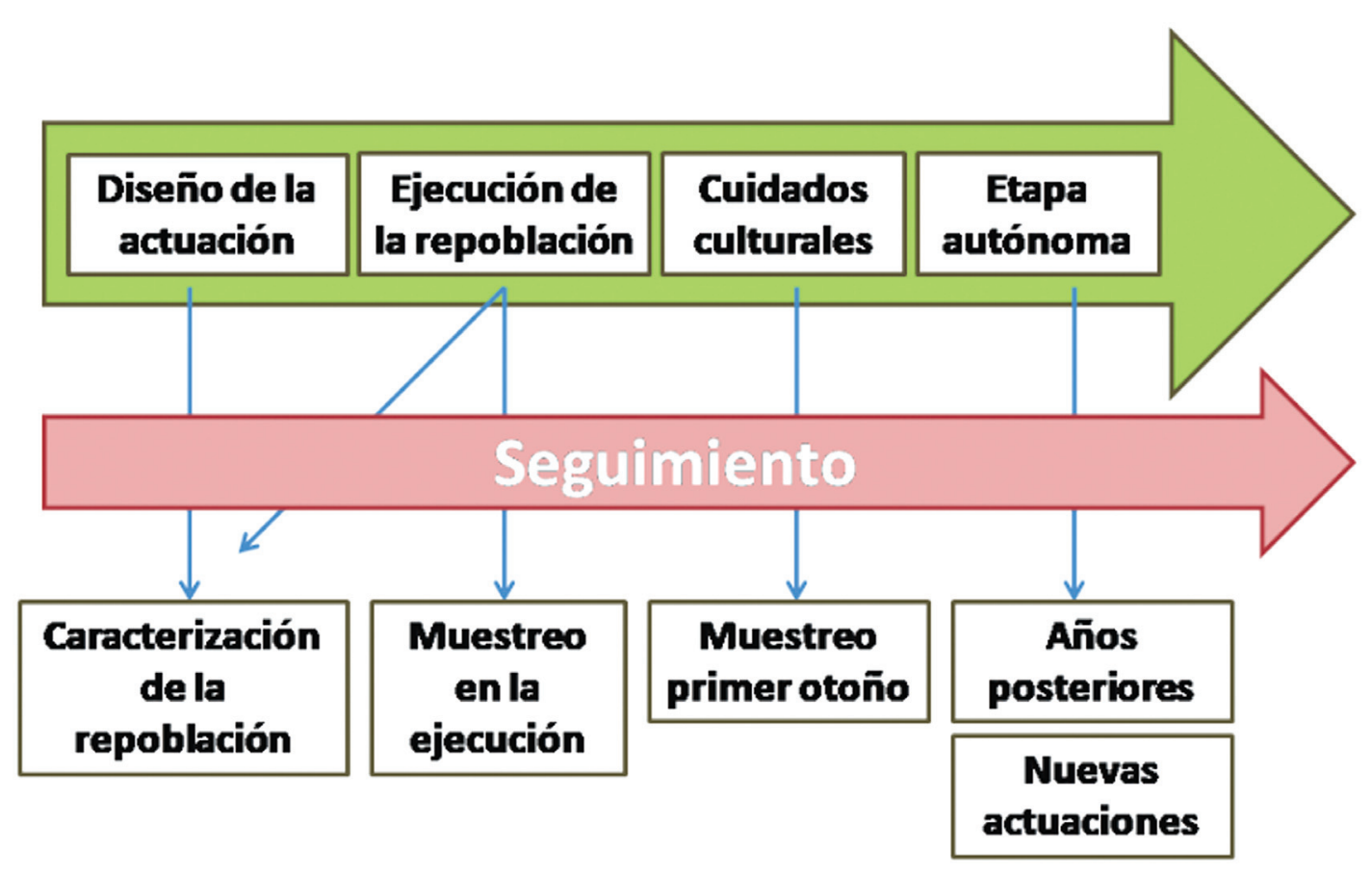

Figura 6. Esquema de las etapas de un proyecto de repoblación y sus relaciones con los momentos claves del seguimiento (Cuevas, 2018).

obtendrá del proyecto de repoblación, siendo necesaria su revisión tras la ejecución o en el caso de realizar nuevas actuaciones.

\subsubsection{Muestreo tras la ejecución}

El primer muestreo se debe realizar una vez concluida la ejecución de la plantación. Este muestreo es muy importante, ya que servirá para tener una referencia precisa de los datos iniciales y previos al primer verano. Cuanto más se retrase el primer muestreo, peor será la calidad del seguimiento en variables ligadas al crecimiento.

\subsubsection{Primer otoño: muestreo post-estival}

La sequía estival es un evento crucial en el mediterráneo para la supervivencia de las plantas, siendo motivo de un aumento de la mortalidad importante. Este muestreo es fundamental si se quiere tener un registro relativamente detallado de la evolución de la plantación en el tiempo.

Su realización será a partir de las primeras lluvias otoñales. Además, permitirá incorporar la información en estudios comparativos inter-proyectos, así como evaluar el efecto de variables ambientales y de ejecución sobre la respuesta del repoblado. Este muestreo también puede resultar imprescindible para dimensionar la actuación de reposición de marras. 


\subsubsection{Años posteriores: muestreo en las etapas de cuidados culturales y autónoma}

Es necesario seguir manteniendo un control durante los siguientes años para ver cómo evoluciona la masa forestal. Según la repoblación se va asentando y ocupando el espacio vegetativo, los cambios son menores y ocurren más despacio, por lo que se podrá disminuir el periodo entre muestreos. Se propone, en función de las etapas, las siguientes frecuencias de muestreo:

- Etapa de cuidados culturales: además del muestreo post-estival, deberán hacerse muestreos el tercer y quinto año desde la plantación.

- Etapa autónoma: los muestreos pueden espaciarse a una frecuencia de cinco años, pudiendo reducirse la frecuencia a partir del segundo muestreo de la etapa autónoma.

\subsubsection{Nuevas actuaciones}

Cuando se produzcan eventos no previstos o nuevas actuaciones que modifiquen sensiblemente la repoblación, es conveniente realizar un nuevo muestreo para evaluar los cambios. Puede ser necesario modificar los rodales de repoblación y reestructurar el seguimiento a partir de este punto.

\subsection{Obtención y almacenamiento de datos}

Un elemento importante a la hora de llevar a cabo la toma de datos es el estadi1lo, el cual debe recopilar información de forma sistemática y exhaustiva (Tablas 2 y 3). Dado que en cada actuación habrá información diferente que recopilar, se podrán emplear distintos modelos de estadillo en función de la situación. Hay que adaptar las plantillas del estadillo para cada situación, con el fin de no incluir información no relevante que pueda generar confusión o un esfuerzo de muestreo innecesario.

Toda esta información será recopilada en una base de datos. Esta base de datos contendrá la información recopilada en el campo en diferentes tablas, que estarán relacionadas entre sí por medio de un modelo relacional (Fig. 7). El empleo de una base de datos relacional evitará la creación de tablas más complejas, separando la información en tantas tablas como sea necesario para que los campos recojan variables únicas.

\subsection{Estimadores}

A la hora de analizar la información, los procedimientos dependerán del tipo de variables que se analicen. En todo caso interesará conocer medias, varianzas y errores. Estos estimadores se calcularán para cada una de las variables, en cada una de las especies medidas en el muestreo. Existen algunas variables que, combinándolas con otras o por medio de modelos e indicadores, permitirán explotar la información con mayor profundidad. 
Tabla 2. Estadillo propuesto para vegetación introducida en este protocolo (Cuevas, 2018).

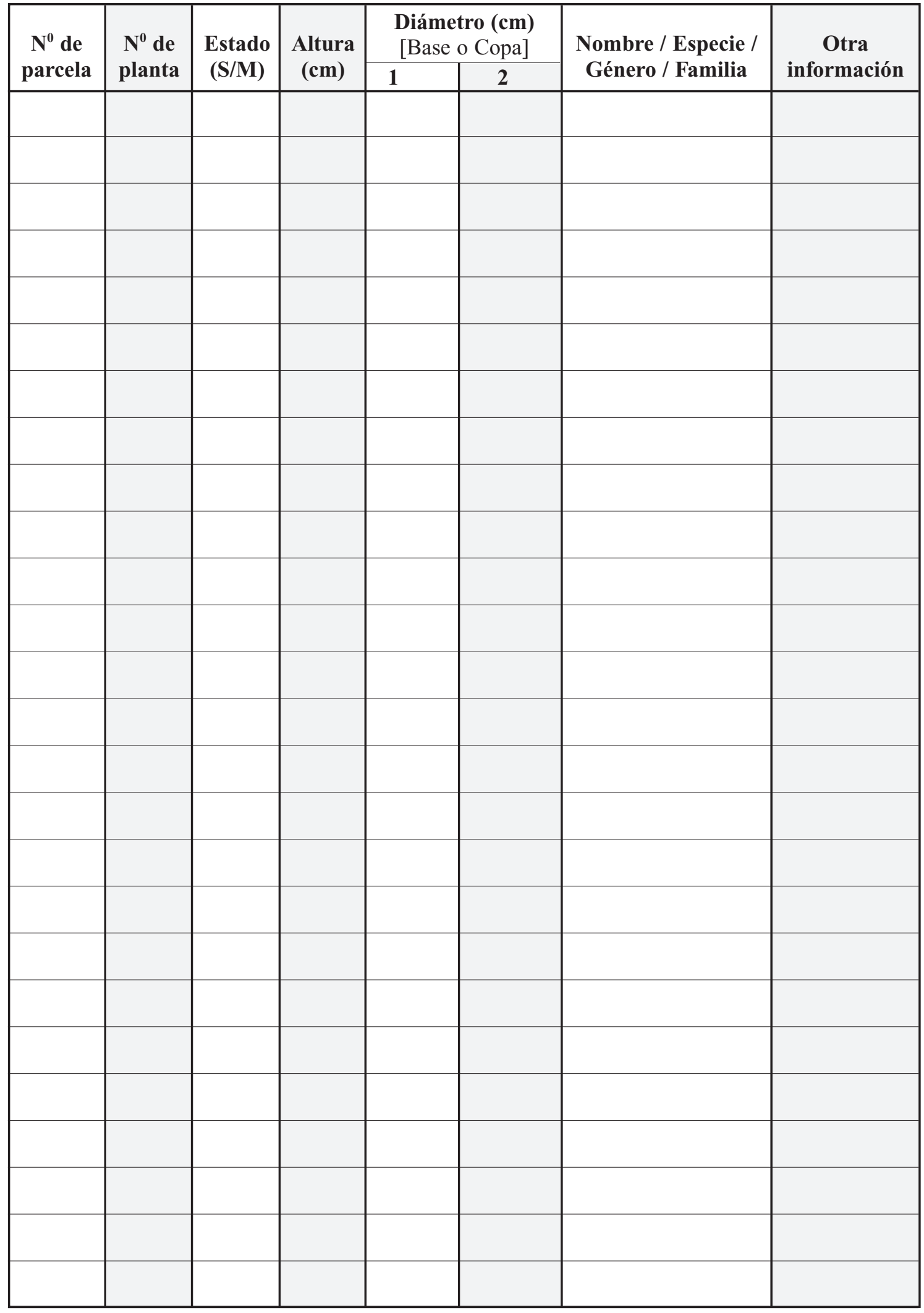


Tabla 3. Estadillo propuesto para vegetación espontánea en este protocolo (Cuevas, 2018).

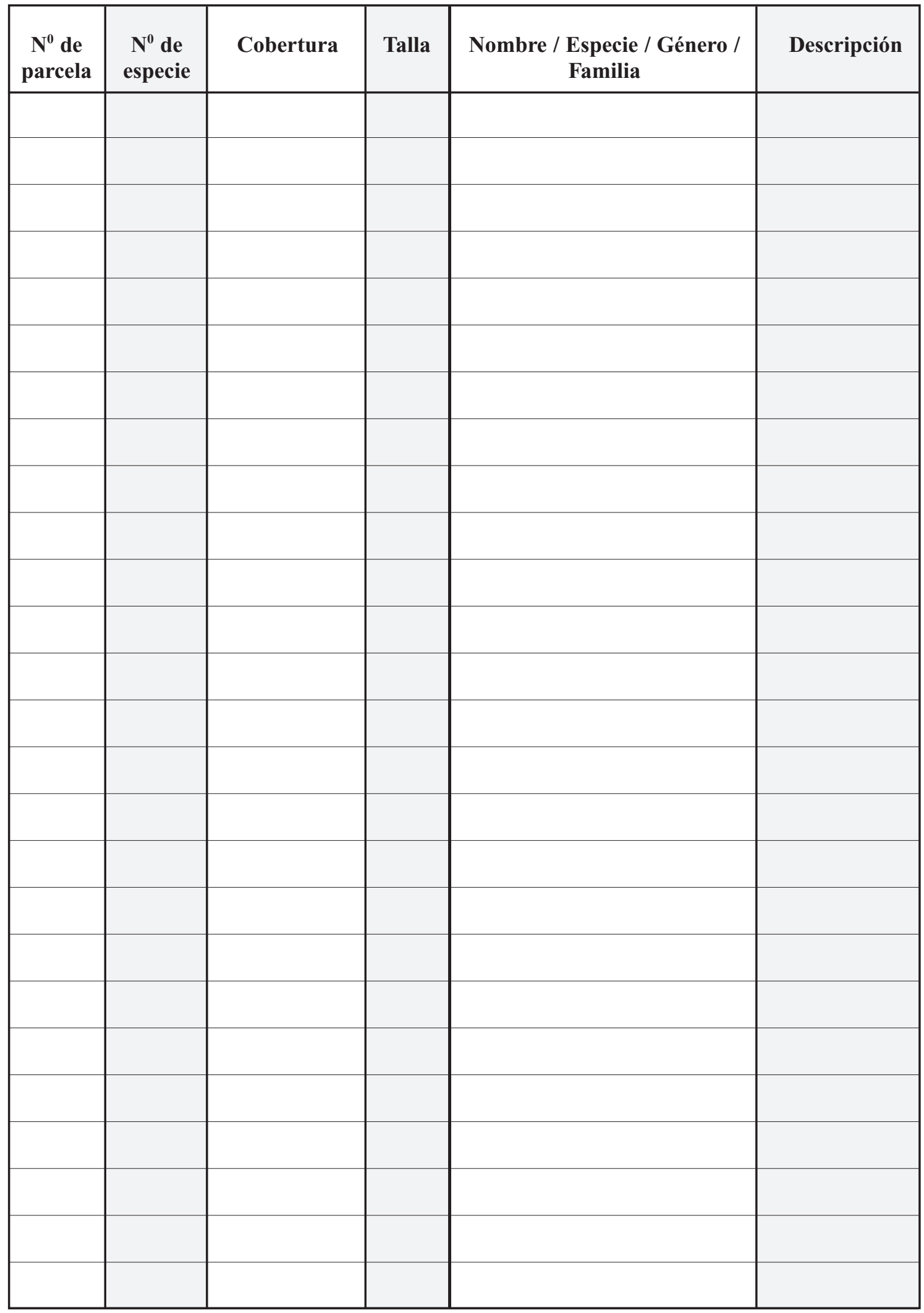




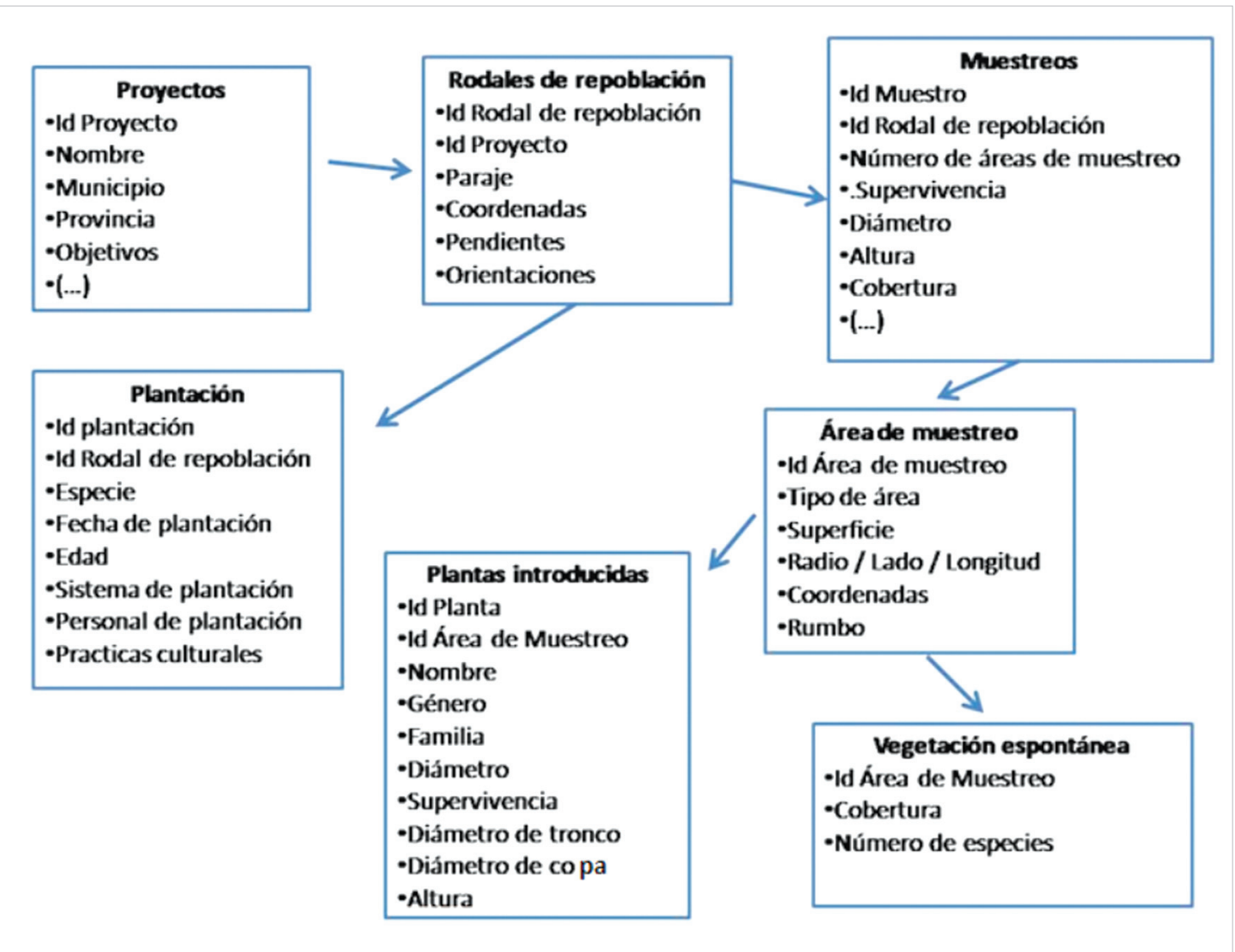

Figura 7. Modelo de datos relacional para la composición de una base de datos adaptado al protocolo (Cuevas, 2018).

Para el caso de la vegetación espontánea, se podrán calcular indicadores de biodiversidad como riqueza de especies e índice de Shannon-Wiener (Del Río et al., 2003).

\section{Conclusiones}

El seguimiento es una labor de interés creciente en el ámbito de la restauración forestal: por una parte, permite aprender de los errores y aciertos de la ejecución de la técnica repobladora y, por otra, extraer información muy valiosa sobre los efectos que en el futuro tendrá la repoblación sobre los sistemas en restauración. Sin embargo, actualmente el seguimiento es una de las mayores carencias detectadas en el desarrollo de proyectos sobre el terreno debido, entre otras causas, a la falta de herramientas técnicas de evaluación adaptadas específicamente a este propósito.

WWF lleva realizando el seguimiento de sus proyectos de restauración forestal desde el año 2009. Sin embargo, se ha encontrado necesario realizar una estandarización en las técnicas y criterios de evaluación para garantizar a lo largo del 
tiempo un seguimiento de calidad de las plantaciones. El protocolo presentado pretende cumplir este objetivo de estandarización para el seguimiento de la vegetación en un proyecto de restauración a corto y medio plazo. El protocolo se centra en optimizar la eficiencia del esfuerzo de muestreo manteniendo una precisión determinada. En este sentido, el protocolo resultante ha demostrado que, en relación con el seguimiento que venía realizando la organización, puede realizarse un monitoreo de mayor calidad de las actuaciones de restauración, con una precisión determinada, sin un coste excesivo.

Además, la experiencia acumulada con estos seguimientos aportará nueva información que permitirá la mejora y retroalimentación del protocolo propuesto. De igual modo, este protocolo representa una oportunidad para que otras entidades públicas o privadas lo adopten y valoren así el éxito de sus restauraciones. A pesar de que este protocolo se centra metodológicamente en los proyectos de WWF, puede tomarse como una recomendación técnica para otros proyectos de restauración. De hecho, su uso podría extenderse a futuros proyectos que emanen de la prevista Estrategia Estatal de Infraestructura Verde y de la Conectividad y Restauración Ecológicas (IVCRE).

No en vano, en los últimos años, la restauración ecológica en España y otras partes del mundo está evolucionando hacia una restauración que asegure la calidad de las intervenciones con proyectos de restauración basados en buenas prácticas, desde la fase de diseño y hasta la evaluación. En este sentido, existen ejemplos de estándares de certificación, como los que está promoviendo la Sociedad de Ecología de la Restauración (SER). En España, algunos ejemplos son los "Estándares de WWF para la Certificación de proyectos de Restauración de Sistemas Forestales", desarrollados en el marco de un grupo de trabajo multidisciplinar integrado por expertos nacionales de diversos campos vinculados a la restauración (Hernández, 2012). También la "Guía Práctica de Restauración Ecológica", recientemente publicada, en la que han participado más de 100 personas del ámbito científico, técnico, de la administración, del sector empresarial y de ONG, con el objetivo de conseguir un documento de consenso que sirva de herramienta metodológica a la futura Estrategia IVCRE (Mola et al., 2018).

La planificación correcta de labores de seguimiento y evaluación es, sin lugar a dudas, uno de los aspectos técnicos en los que podrían mejorar los proyectos de restauración y que recogen todos estos estándares de calidad. Dichas herramientas constituyen un magnífico marco sobre el que desarrollar sistemas de evaluación y seguimiento que permitan mejorar la calidad de las intervenciones y de futuros proyectos de restauración. El protocolo propuesto puede servir como modelo a partir del cual generar otras herramientas extensibles a futuros proyectos.

\section{Bibliografía}

Colomina, D.; Melero, M.; 2016. Recuperando paisajes: un nuevo camino para la restauración. WWF España, Madrid. 
Cuenca, C.; 2014. Análisis de las políticas de restauración forestal en España (1983-2013). Trabajo Fin de Máster. Universidad de Alicante y WWF España, Alicante.

Cuenca, C.; Melero, M.; Cortina, J.; 2016. Análisis de las políticas de restauración forestal en España (1983-2013). Cuad. Soc. Esp. Cienc. For. 42, 61-74. https://doi.org/10.311 67/csef.v0i42.17423

Cuevas, R.; 2018. Protocolo de seguimiento para proyectos de restauración. Universidad Politécnica de Madrid y WWF España, Madrid.

Del Río, M.; Montes, F.; Cañellas, I.; Montero, G.; 2003. Revisión: Índices de diversidad estructural en masas forestales. Invest. Agrar: Sist. Recur. For. 12, 159-176.

Hermosilla, B.; 2016. Elaboración de un protocolo para el muestreo en el seguimiento de repoblaciones forestales. Trabajo Fin de Máster. Universidad de Alcalá, Alcalá de Henares (Madrid).

Hernández, L.; 2012. Estándares de WWF para la Certificación de Proyectos de Restauración de Sistemas Forestales. WWF España, Madrid.

McRoberts, R.; Tomppo, E.; Czaplewski, R.; 2015. Sampling designs for national forest assesment. In: Knowledge reference for national forest assessment. Food and Agriculture Organization of the United Nations (FAO), Rome, pp. 23-40.

Mola, I.; Sopeña, A.; De Torre, R.; 2018. Guía Práctica de Restauración Ecológica. Fundación Biodiversidad del Ministerio para la Transición Ecológica, Madrid

Mueller-Dombois, D.; Ellenberg, H.; 1974. Aims and methods of vegetation ecology. Blackburn Press, Caldwell, New Jersey.

Oliet, J.; De la Hoz, F.M.; Abellanas, B.; Cuadros, S.; Fernández, P.; Zamora, R.; 2005. Manual de ordenación de montes de Andalucía. Consejería de Medio Ambiente. Junta de Andalucía, Sevilla. 